\title{
Effect of vertical quasiperiodic vibrations on the stability of the free surface of an inviscid liquid layer
}

\author{
M. Yagoubi, S. Aniss a and M. Belhaq \\ Laboratory of Mechanics, Faculty of Science Ain Chock, University Hassan II, B.P. 5366, Maarif, Casablanca, Morocco
}

\begin{abstract}
The aim of the present paper is to examine the effect of the vertical quasiperiodic oscillations on the stability of the free surface of an ideal horizontal liquid layer. The quasiperiodic motion considered here is characterized by two incommensurate frequencies $\omega_{1}$ and $\omega_{2}$. The governing system of equations is reduced to a quasiperiodic Mathieu equation. In this situation, using the harmonic balance method developed by Rand et al. $[10,11]$ and Hill's determinants, we determine the marginal stability curves. We show that the quasiperiodic excitation produces a stabilizing or a destabilizing effect and is strongly depending on the ratio of the frequencies.
\end{abstract}

\section{Introduction}

The Faraday instability is a parametric generation of standing waves on the free surface of a liquid subjected to vertical vibrations. This phenomenon has been studied by Faraday [1]. The initially flat free surface of the fluid becomes unstable at a critical intensity of the vertical vibrations of the whole system. A pattern of standing waves is then formed at the fluid surface. Faraday has observed that the typical response is subharmonic, i.e the wave frequency is half the frequency of the excitation. Subsequently, the problem was investigated by Matthiessen [2, 3]. He has found in his experiment that the vibrations were synchronous. These discrepancies have led Lord Rayleigh $[4,5]$ to carried out a further series of experiments which have supported Faraday's view. Later on, Benjamin and Ursell [6], have analyzed theoretically the linear problem for ideal liquids enclosed in a container, vibrating sinusoidally in the vertical plane, and have shown that the governing equations can be reduced to a system of Mathieu equations which allow harmonic as well as subharmonic solutions. A detailed experimental study of the various patterns on a viscous fluid has been performed by Edwards and Fauve [7] who have used oscillations with one frequency as well as oscillations with two frequency. The linear stability analysis of the Faraday instability on an arbitrarily deep layer of a viscous fluid has been performed by Kumar and Tuckerman [8] and a discussion on the mechanism of the wave number selection in the Faraday instability on high-viscous fluids have been done by Kumar [9]. For a review, see [10 - 15].

In contrast to the previous works using the standard periodic oscillations, the present work focuses attention on the influence of a quasiperiodic excitation on the stability of the free surface of an horizontal inviscid liquid layer under vertical quasiperiodic displacement having two incommensurate frequencies. Following [6], we reduce the linear problem to a linear quasi-periodic Mathieu oscillator. Note that in this case, Floquet theory cannot be applied to deter- mine a stability criterion. However, the works by Rand et al. $[10,11]$ provide a stability chart for the quasiperiodic Mathieu oscillator. This result is used to investigate the quasiperiodic parametric instability in our specific physical problem and to determine the marginal stability curves as a function of the various parameters of the problem.

\section{Formulation}

Consider a liquid layer of a depth $h$ below the free surface, see Fig. 1. The fluid layer is submitted to vertical quasiperiodic motion according to the law of displacement.

$$
z(t)=\alpha_{1} \cos \left(\omega_{1} t\right)+\alpha_{2} \cos \left(\omega_{2} t\right)
$$

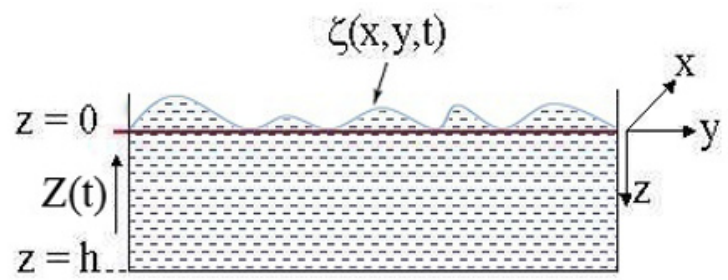

Fig. 1. The hydrodynamical system.

where $\omega_{1}$ and $\omega_{2}$ are two incommensurate frequencies. The coefficients $\alpha_{1}$ and $\alpha_{2}$ are the amplitudes of motion. Therefore, the fluid layer is submitted to two volumic forces: the gravitational force $\rho \mathrm{g}$ and the quasiperiodic one, $-\rho\left[\alpha_{1} \omega_{1}^{2} \cos \left(\omega_{1} t\right)+\alpha_{2} \omega_{2}^{2} \cos \left(\omega_{2} t\right)\right] \mathbf{k}$. We denote by $\mathbf{k}$, the unit vector upward. We consider in this work the case of an ideal fluid in which viscosity and effects due to wetting of the walls are neglected. Under these assumptions, the linear system of the governing equations, corresponding to the perturbation of the equilibrium state, is given by the following Navier-Stokes equations.

a e-mail: saniss@hotmail.com

$\nabla \cdot \mathbf{V}=0$ 


$$
\frac{\partial \mathbf{V}}{\partial t}=-\frac{1}{\rho} \nabla p+\left(g-\alpha_{1} \omega_{1}^{2} \cos \omega_{1} t-\alpha_{2} \omega_{2}^{2} \cos \omega_{2} t\right) \mathbf{k},
$$

where $u(x, y, z, t), v(x, y, z, t), w(x, y, z, t)$ are the components of the velocity field and $p(x, y, z, t)$ is the pressure. The density $\rho$ is assumed to remain constant during the motion. If the motion was originally started from rest, there is a velocity potentiel $\phi(x, y, z, t)[12]$ such that $(u, v, w)=$ $\left(\frac{\partial}{\partial x}, \frac{\partial}{\partial y}, \frac{\partial}{\partial z}\right) \phi$, and therefore the equations of motion have the integral

$$
\begin{array}{r}
\frac{p}{\rho}+\frac{\partial \phi}{\partial t}+\frac{1}{2}\left(u^{2}+v^{2}+w^{2}\right)-\left(g-\alpha_{1} \omega_{1}^{2} \cos \omega_{1} t\right. \\
\left.-\alpha_{2} \omega_{2}^{2} \cos \omega_{2} t\right) z=F(t),
\end{array}
$$

where $F(t)$ is independent of $x, y, z$, and may be equal to zero. Equation (2) becomes

$$
\frac{\partial^{2} \phi}{\partial x^{2}}+\frac{\partial^{2} \phi}{\partial y^{2}}+\frac{\partial^{2} \phi}{\partial z^{2}}=0
$$

Using the dynamic boundary condition at the interface, we obtain the equation of the free surface

$$
\begin{aligned}
\frac{\sigma}{\rho}\left(\frac{\partial^{2} \zeta}{\partial x^{2}}+\frac{\partial^{2} \zeta}{\partial y^{2}}\right)+\frac{\partial \phi}{\partial t}+ & \frac{1}{2}\left(u^{2}+v^{2}+w^{2}\right)-\left(g-\alpha_{1} \omega_{1}^{2}\right. \\
& \left.\cos \omega_{1} t-\alpha_{2} \omega_{2}^{2} \cos \omega_{2} t\right) \zeta=0
\end{aligned}
$$

where $z=\zeta(x, y, t)$ and $\sigma$ is the surface tension. The kinematical surface condition [12] is

$$
\frac{D}{D t}(\xi(x, y, t)-z) \equiv \frac{\partial \xi}{\partial t}+u \frac{\partial \xi}{\partial x}+v \frac{\partial \xi}{\partial y}+w=0
$$

The normal velocities at the walls and at the base of the vessel, relative to the moving axes, are considered equal to zero. Then

$$
\begin{aligned}
& \frac{\partial \phi}{\partial n}=0 \\
& \frac{\partial \phi}{\partial z}=0
\end{aligned}
$$

After that equations (7) and (6) are written as,

$$
\begin{gathered}
\frac{\partial \zeta}{\partial t}=w=\frac{\partial \phi}{\partial z} \quad \text { at } \quad z=0 \\
\frac{\sigma}{\rho}\left(\frac{\partial^{2} \zeta}{\partial x^{2}}+\frac{\partial^{2} \zeta}{\partial y^{2}}\right)+\left(\frac{\partial \phi}{\partial t}\right)_{z=0}+\frac{1}{2}\left(u^{2}+v^{2}+w^{2}\right)-(g \\
\left.-\alpha_{1} \omega_{1}^{2} \cos \omega_{1} t-\alpha_{2} \omega_{2}^{2} \cos \omega_{2} t\right) \zeta=0
\end{gathered}
$$

\section{Quasiperiodic Mathieu equation}

The boundary conditions, (8) and (10), show that $\phi, \zeta$ and $\left(\frac{\partial^{2} \zeta}{\partial x^{2}}+\frac{\partial^{2} \zeta}{\partial y^{2}}\right)$ can each be expanded in terms of the complete orthogonal set of eigenfunctions $S_{m}(x, y)$, where

$$
\left(\frac{\partial^{2}}{\partial x^{2}}+\frac{\partial^{2}}{\partial y^{2}}+k_{m}^{2}\right) S_{m}(x, y)=0
$$

This system has non trivial solutions only when $k_{m}^{2}$ is an eigenvalue [15]. We decompose the solutions of the equation (11) on the basis of eigenfunctions $S_{m}$. We obtain the following amplitude equation

$$
\begin{array}{r}
\frac{d^{2} A_{m}}{d t^{2}}+k_{m} \tanh \left(k_{m} h\right)\left(\frac{k_{m}^{2} \sigma}{\rho}+g-\alpha_{1} \omega_{1}^{2} \cos \omega_{1} t\right. \\
\left.-\alpha_{2} \omega_{2}^{2} \cos \omega_{2} t\right) A_{m}=0
\end{array}
$$

In this work, we assume that

$$
\alpha_{1} \omega_{1}^{2}=\alpha_{2} \omega_{2}^{2}
$$

The amplitude equation (13) becomes

$$
\begin{array}{r}
\frac{d^{2} A_{m}}{d t^{2}}+k_{m} \tanh \left(k_{m} h\right)\left(\frac{k_{m}^{2} \sigma}{\rho}+\right. \\
+\cos \omega_{1} \omega_{1}^{2}\left(\cos \omega_{1} t\right. \\
+A_{m}=0
\end{array}
$$

The change of variable $\tau=\omega_{1} t$ reduces Eq.(15) to the quasiperiodic Mathieu equation studied by Rand et al. [10, $11]$.

$$
\frac{d^{2} A}{d \tau^{2}}+[\delta+\epsilon(\cos \tau+\cos \omega \tau)] A=0
$$

where

$$
\begin{gathered}
\delta=\frac{k_{m} \tanh \left(k_{m} h\right)}{\omega_{1}^{2}}\left(\frac{k_{m}^{2} \sigma}{\rho}+g\right), \\
\epsilon=-k \tanh (k h) \alpha_{2} \omega^{2}, \quad \omega=\frac{\omega_{2}}{\omega_{1}} .
\end{gathered}
$$

As noticed before, the Floquet theory cannot be used to determine solutions of Eq. (16). Following Rand et al. [10, 11], we use the harmonic balance method to determine the marginal stability curves by means of expansion

$$
A(\tau)=\sum_{n=0}^{\infty} \sum_{m=-\infty}^{\infty}\left[C_{n m} \cos \left(\frac{n+m \omega}{2} \tau\right)+D_{n m} \sin \left(\frac{n+m \omega}{2} \tau\right)\right]
$$

Approximate results are obtained by a truncation of the infinite sums in Eq. (17) and then replaced by sums from 0 to $N$ for $n$ and from $-N$ to $N$ for $m$, respectively. In the case, $N=1(n=0,1 ; m=-1,0,1)$, Eqs. (16) and (17) allows us to obtain two homogenous algebraic systems in $C_{n m}$ and $D_{n m}$ which verify: $C_{-n,-m}=C_{n, m}$ and $D_{-n,-m}=-D_{n, m}$. The first system in $C_{n m}$ is in the form

$$
\left\{\begin{array}{l}
\left(\delta-\frac{\omega^{2}}{4}+\frac{\epsilon}{2}\right) C_{0,1}=0 \\
\delta C_{0,0}=0 \\
\left(\delta-\frac{(1-\omega)^{2}}{4}\right) C_{1,-1}+\epsilon C_{1,1}=0 \\
\left(\delta-\frac{1}{4}+\frac{\epsilon}{2}\right) C_{1,0}=0 \\
\left(\delta-\frac{(1+\omega)^{2}}{4}\right) C_{1,1}+\epsilon C_{1,-1}=0
\end{array}\right.
$$


The second system in $D_{n m}$ is given by

$$
\left\{\begin{array}{l}
\left(\delta-\frac{\omega^{2}}{4}+\frac{\epsilon}{2}\right) D_{0,1}=0 \\
\left(\delta-\frac{(1-\omega)^{2}}{4}\right) D_{1,-1}=0 \\
\left(\delta-\frac{1}{4}-\frac{\epsilon}{2}\right) D_{1,0}=0 \\
\left(\delta-\frac{(1+\omega)^{2}}{4}\right) D_{1,1}=0
\end{array}\right.
$$

Coupling these two systems, we obtain the following system in $C_{n, m}$

$$
\left(\begin{array}{ccccc}
C_{11} & 0 & 0 & 0 & 0 \\
0 & C_{22} & C_{23} & 0 & 0 \\
0 & C_{32} & C_{33} & 0 & 0 \\
0 & 0 & 0 & C_{44} & 0 \\
0 & 0 & 0 & 0 & C_{55}
\end{array}\right)\left(\begin{array}{c}
C_{0,0} \\
C_{1,-1} \\
C_{1,1} \\
C_{0,1} \\
C_{1,0}
\end{array}\right)=0
$$

where

$$
\begin{gathered}
C_{11}=\delta, \quad C_{22}=\delta-\frac{(1-\omega)^{2}}{4}, \\
C_{23}=C_{32}=\epsilon, \quad C_{33}=\delta-\frac{(1+\omega)^{2}}{4}, \\
C_{44}=\delta-\frac{\omega^{2}}{4}+\frac{\epsilon}{2}, \quad C_{44}=\delta-\frac{1}{4}+\frac{\epsilon}{2}
\end{gathered}
$$

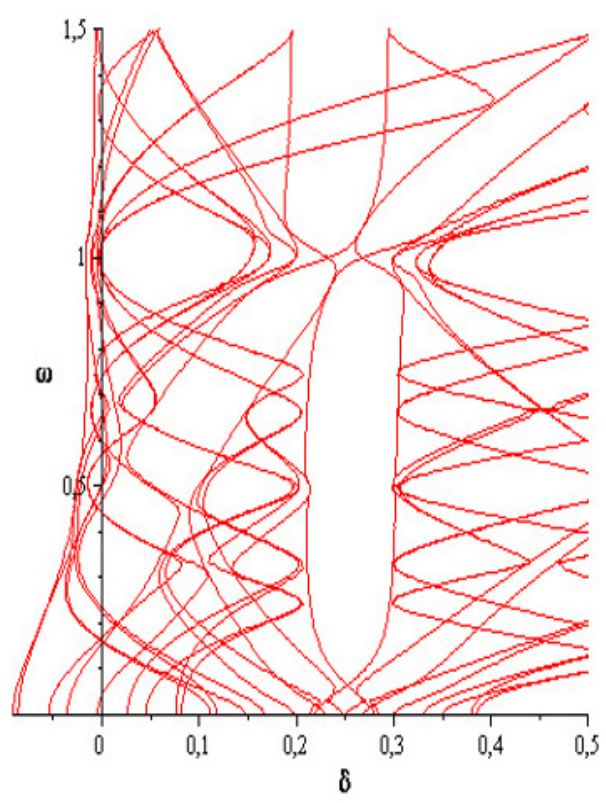

Fig. 2. Stability chart of the quasiperiodic Mathieu equation in the plane $(\delta, \omega)$ for $N=4, \epsilon=0.1$.

The system (20) have non-trivial solution if its determinant vanishes. For each $N$, the dimension of this system is $2 N^{2}+2 N+1$. For the case $N=4$ considered in the current paper, the corresponding system dimension is equal to 41 . Nevertheless, the analysis is facilitated by putting the system in upper triangular form. We show in Fig. 2 in the plane $(\delta, \omega)$ when $\epsilon=0.1$, the stability chart as obtained by Rand et al. [10, 11].The marginal stability curves, $\alpha_{2}(k)$, are determined numerically by fixing the dimensionless frequency, $\omega_{1}$, the frequency ratio, $\omega$, the depth, $h$ and the fluid characteristics studied (pure water at $16^{\circ} \mathrm{C}$ ). Hereafter, we focus attention on the curves corresponding to the variation of the vibration amplitude, $\alpha_{2}$, versus the wave number, $k$.

\section{Results and discussion}

\subsection{Influence of the excitation frequency $\omega_{1}$}

In Fig. 3, we present for $h=0.1 \mathrm{~cm}, \omega=\sqrt{2}$ and for different values of $\omega_{1}$, the marginal stability curves in term of amplitude vibration, $\alpha_{2}$, and wave number, $k$. We note that for small values of $\omega_{1}$ the system is stable and for large values, two unstable regions appear for $\omega_{1}=50 \mathrm{~Hz}$. Also, one can see that other regions of instability arise for $\omega_{1}=$ $50 \mathrm{~Hz}$. Otherwise, the wave number corresponding to the resonance in the principal regions of instability increases with the frequency $\omega_{1}$.
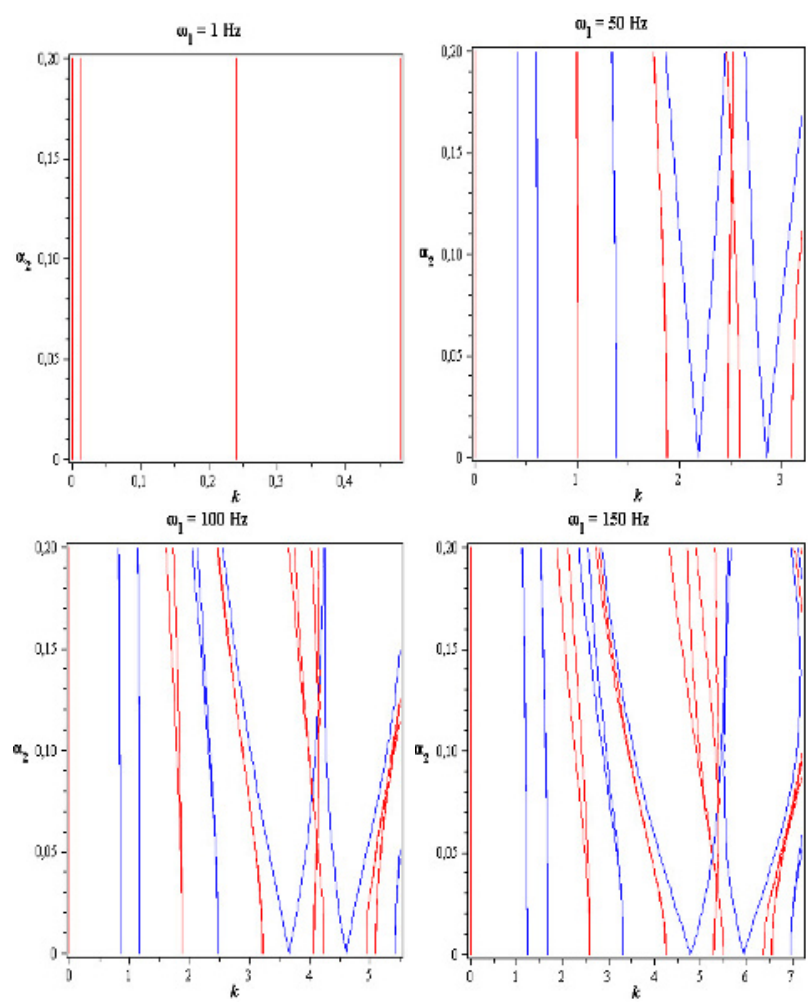

Fig. 3. Marginal stability curves for $h=0.1 \mathrm{~cm}, \omega=\sqrt{2}$ and for different values of the frequency, $\omega_{1}$.

\subsection{Influence of frequencies ratio $\omega$}

In Fig. 4, we report results corresponding to the influence of the irrational frequencies ratio on the instability regions in the plane $\alpha_{2}, k$ for $\omega_{1}=50 \mathrm{~Hz}$ and $h=0.1 \mathrm{~cm}$. In this 
figure, we observe that the instability regions arise gradually when the irrational frequencies ratio, $\omega$, increases. Finally, remark that the wave number, $k$, corresponding to the first resonance does not depend on the frequency ratio $\omega$.
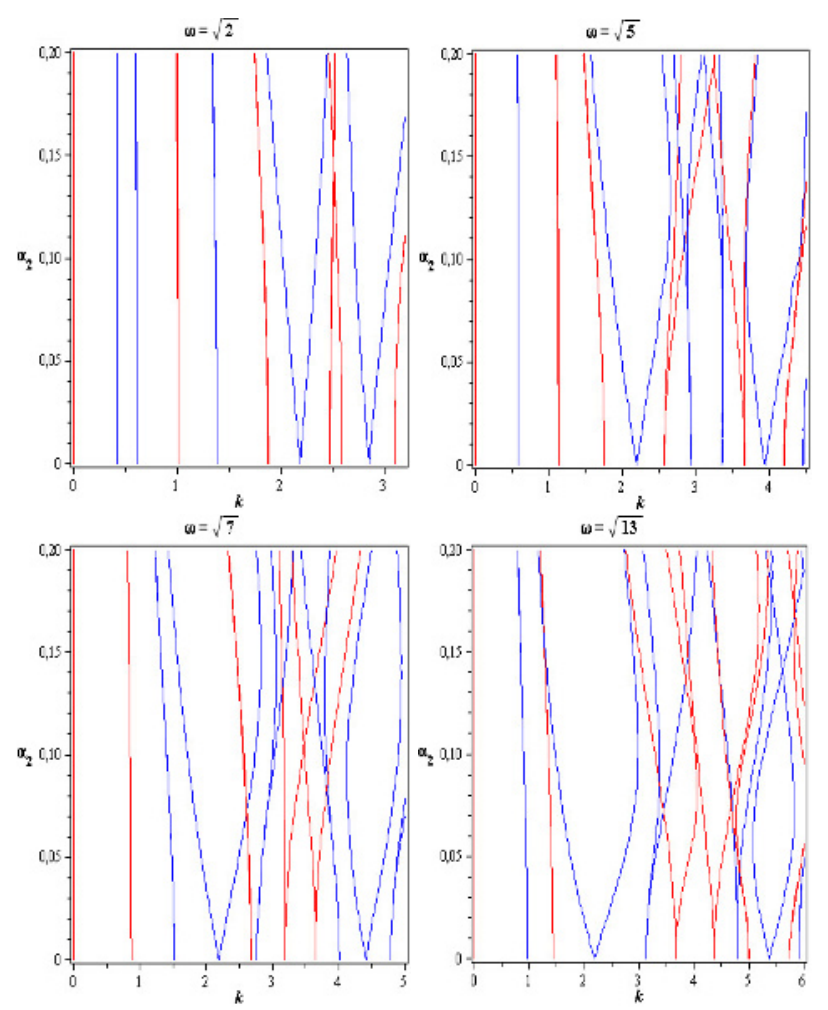

Fig. 4. Marginal stability curves for $h=0.1 \mathrm{~cm}, \omega_{1}=50 \mathrm{~Hz}$ and for different values of $\omega$.

\section{Conclusion}

In this work, we have studied the effect of vertical quasiperiodic oscillations on the stability of a free surface of an ideal fluid layer. The linear equations of motion are reduced to a damped quasiperiodic Mathieu equation. It was shown that oscillations with two incommensurate frequencies have a pertinent influence on the diagram of instability in the plane amplitude vibrations and wave number.

\section{References}

1. M. Faraday, Philos. Trans. R. Soc. Lond, 121, (1831) 319-340.

2. L. Matthiessen, Ann. Phys., Lpz, 134, (1868) 107-117.

3. L. Matthiessen, Ann. Phys., Lpz, 141, (1870) 375-393.

4. L. Rayleigh, Phil. Mag., 15, (1883) 235.

5. L. Rayleigh, Phil. Mag., 16, (1883) 50.

6. T. B. Benjamin, F. Ursell, Proc. Roy. Soc. Lond. A, 255, (1954) 505-515.

7. W.S. Edwards and S. Fauve, Phys. Rev., 47, (1993) 788791.

8. K. Kumar and L. S. Tuckerman, J. Fluid Mech., 279, (1994) 49-68.
9. S. Kumar, Phys. Rev., 62, (2000) 1416.

10. R. Rand, R. R. Zounes and R. Hastings, Nonlinear Dynamics: The Richard Rand 50th Anniversary volume, World Scientific, Singapore, (1997) chap. 9.

11. R. S. Zounes and R. Rand, SIAM J. Appl. Math., 58, (1998) 1094-1115.

12. H. Lamb, Hydrodynamics, 6th ed. Cambridge University Press (1932).

13. T. Boulal, S. Aniss, M. Belhaq and R. Rand, Phys. Rev., 76, (2007) 56320.

14. T. Boulal, S. Aniss, M. Belhaq and A. Azouani, Int. J. Non-Linear Mech., 43, (2008) 852-857.

15. R. Courant and D. Hilbert, Methoden der mathematishen Physik, 2nd ed. Berlin: Springer, (1931). 